\title{
Individuelle Förderung bildnerischer Kompetenzen im Kunstunterricht ${ }^{2}$
}

\author{
Individual promotion of visual skills in art classes
}

\author{
Stefan Mayer ${ }^{3}$ \\ Data de Submissão: 11 jul. 2020 \\ Data de Aprovação: 25 ago. 2020. \\ Data de Publicação: 30 dez. 2020
}

\begin{abstract}
ZUSAMMENFASSUNG: In diesem Aufsatz wird das Thema der Individualisierung des Unterrichts durch individuelle Unterstützung im Kunstunterricht erörtert. Der Text enthält drei Beispiele dafür, wie sich künstlerische Aktivitäten auf Basis der individuellen Unterstützung entwickeln können. In den Beispielen wird erläutert, dass individuelle Unterstützung nicht primär ein Training der Feinmotorik ist, sondern ein Unternehmen, das nicht nur langfristiges ästhetisches Interesse wecken und fördern, sondern auch Talente entdecken und entwickeln kann.
\end{abstract}

Schlüsselwörter: Individualisierung. Individuelle Unterstützung. Kunstkurse. persönliche Disposition.
ABSTRACT:: This essay discusses the issue of individualization of teaching, through individual support in art classes. The text presents three examples of how art activities can rely on this type of individual support. In the examples, it is discussed how individual support is not a training of fine motor skills, but, rather, an enterprise that can not only awaken and promote longterm aesthetic interest, but discover and develop talents.

Keywords: Individualization. Individual Support. Art Classes. Motor skills.

\section{EINFÜHRUNG}

Die kontrovers diskutierte Frage nach einer Individualisierung hat den Kunstunterricht erreicht. Ist ein individuelles Fördern nicht eine Überlastung der Kunstpädagogin bzw. des Kunstpädagogen und bedeutet eine individuelle Förderung - neben der omnipräsenten Kompetenzorientierung - nicht nur noch eine Sorge mehr? Welche Rolle kann eine individuelle Förderung im Kunstunterricht spielen und wie kann sie aussehen?
Die aktuelle kunstpädagogische Forschung rekurriert bei einer Förderung individueller bildnerischer Prozesse, motiviert durch die Unmöglichkeit eines normativen Kunstbegriffs, bevorzugt auf das Feld der Zeichnung. Eine Strategie, die sich seit Kerschensteiner (Kerschensteiner 1905), der auf die Zeichnung als zentralen Indikator setzte, grundlegend nicht geändert hat.

Zumindest in den aktuellen bayerischen Kunstlehrplänen ist praktisch nichts von einer - wie auch immer gearteten - Individualisierung des Unterrichts zu sehen.

\footnotetext{
${ }^{1}$ CC BY Attribution: Dies ist ein Open-Access-Artikel, der unter den Creative Commons Attribution-Lizenzbedingungen vertrieben wird. Die Lizenz erlaubt die Verwendung, Verbreitung und uneingeschränkte Vervielfältigung in jedem Medium, sofern die Originalquellen gutgeschrieben werden.

2 Ursprünglich in der Zeitschrift veröffentlicht BDK Mitteilungen (Fachzeitschrift des BDK (Bund Deutscher Kunsterzieher) Fachverband für Kunstpädagogik) N. 4/2107, p. 15-19, und vom Autor an die JOSSHE v.3, n.2, 2020.

3 Promotion in Kunstpädagogik (2011) an der Universität Augsburg. Chefredakteur des Journal of Social Sciences, Humanities and Research in Education - JOSSHE - (Journals für Sozial-, Geistes- und Bildungsforschung); Mitherausgeber der Zeitschrift Kunst und Unterricht und Mitwirkender an der Kammerlohr-Reihe. Beauftragter Professor an der Justus Liebig Universität Würzburg. DOI: https://orcid.org/0000-0001-5962-2726.
} 
Allerdings findet sich im neuen Bayerischen "Lehrplan+" das (explizit feinmotorische) Zeichnen als eine Konstante, die sich durch alle Jahrgänge zieht und auf diese Weise omnipräsent im Unterrichtsalltag sein wird. Dabei ist eine individuelle Förderung nicht ein Training feinmotorischer Fähigkeiten, sondern zunächst ein in die Breite gehendes Unternehmen, das durchaus im Schulalltag auf einfache Weise Raum finden kann; dazu drei Beispiele.

Abb. 1: Die beiden Rennmaschinen vor der Tribüne, die Möglichkeiten und Grenzen von Wellpappe als Rennmaterial auslotend, ausgedacht und realisiert in einem Halbjahresprojekt eines Kunstadditums.

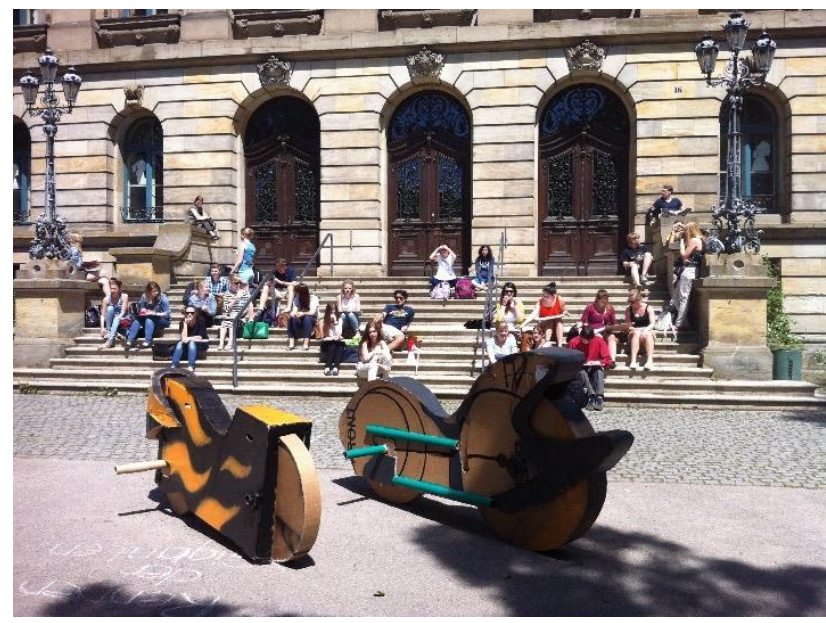

Quelle: Mayer_Rennen_01

Ausgangspunkt für ein Halbjahresprojekt im Bereich „Objekt und Design“ war die elfte Klasse eines musischen Gymnasiums in Bayreuth, sie befasste sich damit, ein "Rennen“ zu organisieren. Einziger Rahmen des bewusst sehr offen gehaltenen Themas war die Materialvorgabe, es sollte ausschließlich Wellpappe verwendet werden (Abb. 1). Die Schülerinnen und Schüler waren gewohnt, Arbeitsaufträge in der Gruppe zu besprechen, was eine Orientierung im Themenbereich und das Ausloten möglicher Wege rasch vonstatten gehen ließ. Umsetzbar erschienen prinzipiell Lösungen im Modellbereich oder in benutzbarer Größe vierrädrig oder zweirädrig.

Eine Recherche im Kunst- und Designbereich steckte einen zusätzlichen Horizont ab. Nachdem grundsätzlich von Piloten steuerbare Vehikel präferiert wurden, ging die Gruppe daran sich mit dem Material bekannt zu machen: Was ist ein realistisches Ziel, das mit den begrenzten Mitteln in einem überschaubaren Zeitrahmen erreicht werden kann?
Die Gruppe beschloss, sich in zwei konkurrierenden Teams aufzuteilen, die mit einer Art Draisinen gegeneinander antreten wollten (Abb. 2). Eine Überraschung für die Teilnehmenden boten die vielen verschiedenen Aufgabenfelder, die sich bei vertiefender Beschäftigung mit der Themenstellung auftaten.

Abb. 2: Die beiden Kontrahenten des „Rennen auf Wellpapperädern" vor dem Start, Halbjahresprojekt eines Kunstadditums.

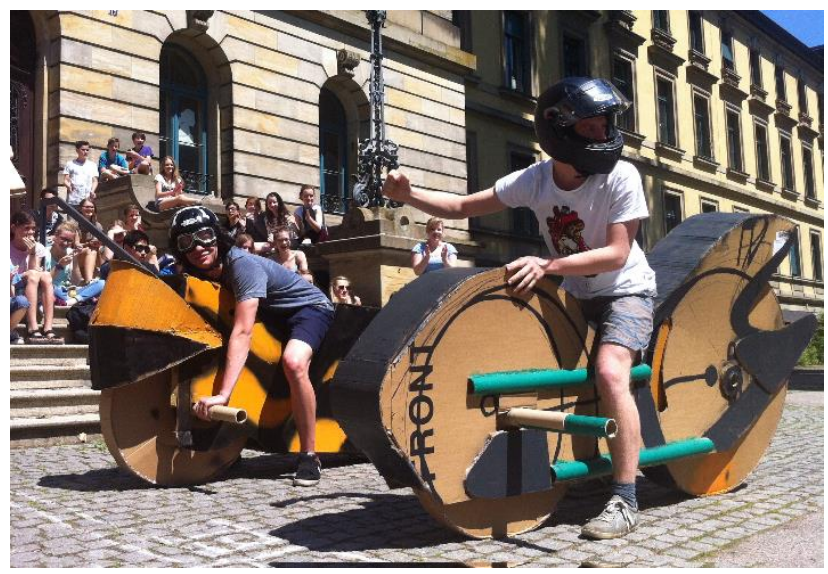

Quelle: Mayer_Rennen_02

Natürlich mussten zunächst grundsätzliche Probleme gelöst werden: Wie etwa lenkt ein Wellpappe-„,Fahrrad“? Wie können Räder gebaut und gelagert werden, nur mit Wellpappe? (Abb. 3) Es ging allerdings nicht nur ums Erfinden, Planen und Bauen, oder ums Zeichnen und Entwerfen, sondern auch um das Promoten, das Erstellen der Plakate und des "Making-offs", um das Festlegen einer Rennstrecke, das Organisieren einer Veranstaltung und darum, letztlich eine Performance durchzuführen.

Abb. 3: Die beiden Rennmaschinen vor dem Start, Kieselsteine in den Rädern erzeugen Lärm und sollen den Gegner einschüchtern, ein „Rennen auf Wellpapperädern“, ausgedacht und realisiert als Halbjahresprojekt eines Kunstadditums.

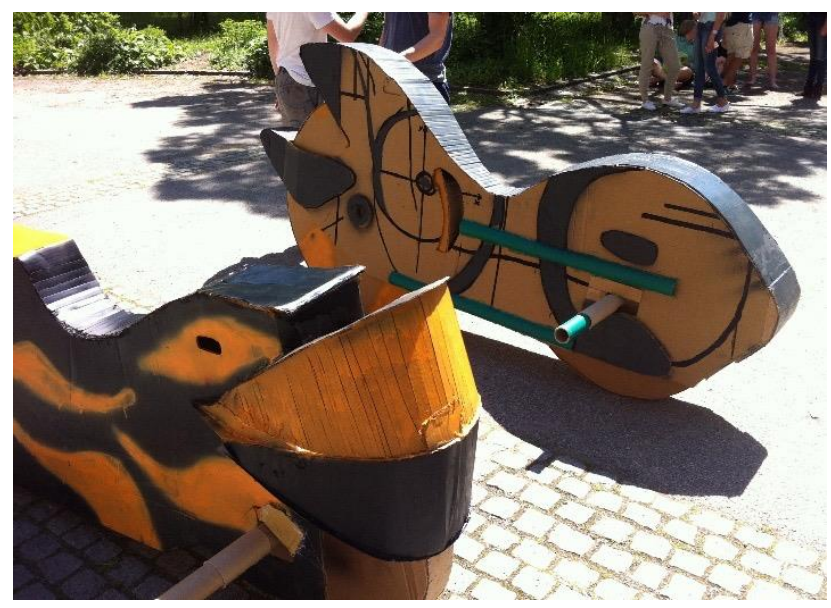


Quelle: Mayer_Rennen_03

Die offene Themenstellung bedeutete angesichts der fehlenden Aufgabenstruktur und der Vielfalt der bildnerischen Tätigkeitsfelder eine tendenzielle Überforderung der Schülerinnen und Schüler. Es konnte die folgerichtig erscheinende Reaktion beobachtet werden, dass sich die Teilnehmenden überlegten, wie sie sich in das Projekt einbringen könnten; und zwar nicht nur mit dem, was sie können, nicht nur dort, wo ihnen etwas einfallen könnte, sondern vor allem mit dem, was ihnen leichtfällt, wozu sie sich veranlagt fühlten. Die Jugendlichen versuchten in gewisser Weise das Thema auf ihre Seite zu ziehen, dorthin wo sie eine persönliche Veranlagung vermuteten.

„Um das ästhetische Interesse nachhaltig zu wecken und zu fördern und damit zugleich Begabungen zu entdecken und zu entfalten, muss es Aufgabe des Kunstunterrichts sein, den Schülerinnen und Schülern möglichst vielfältige Bereiche bildnerischer Produktion und Rezeption verfügbar zu machen." (Kirchner 2008, S. 1)

Offene Aufgaben gelten gemeinhin als Förderinstrument divergenten Denkens, können aber durchaus zu einem Hinterfragen persönlicher Dispositionen führen, einer Reflexion der eigenen Voraussetzungen und persönlicher Begabungen. Bildnerische Begabungen sind nach einer Definition des Kunstpädagogen Frank Schulz, Dispositionen, die in der Sprache der Formen und Farben innere Zustände sichtbar machen und ein entsprechendes Verhältnis zur Wirklichkeit klären (Schulz 2015, S. 431f.).

Angesichts der Bandbreite künstlerischer Strategien liegt es nahe, über Farbe und Form hinaus zu denken, so dass nicht das Sichtbarmachen, sondern das „in eine Form bringen“, das „Form Finden" in den Mittelpunkt bildnerischer Begabung rückt.

\section{Kunstclub}

Kunstlehrende haben sich schon immer um ihre begabten Schülerinnen und Schüler, besonders um die, die zeichnen und malen, gekümmert. Es waren immer diejenigen, die dadurch auffielen, dass sie etwas wollten, die von den Kunstlehrenden beiseite genommen und mit besonderen Gestaltungsaufträgen bedacht wurden. Heute sieht der Begabungsforscher Roth darin wesentliche
Ansätze zu einer effektiven Förderung: Es handelt sich um Angebote zur persönlichen Entwicklung, um Anregungen zur stetigen Ermutigung und um das Schaffen von musischen Freiräumen (Roth 2015, S. 74). Besonders Letzteres geht manchmal ganz einfach und ereignet sich zum Beispiel an informellen Ateliertagen, Maltagen oder wie bei diesem Beispiel in einem informellen Kunstclub. Seit fast drei Jahren treffen sich an einem Samstag im Monat Schülerinnen und Schüler aller Jahrgangsstufen eines Bayerischen Gymnasiums, die Lust aufs Zeichnen, Malen und Gestalten haben. Die Zeit zwischen den „Maltagen“ findet ein loser Austausch über diverse soziale Netzwerke statt. Gleichgesinnte haben so die Möglichkeit, sich kennen zu lernen und über ihre bildnerische Arbeit zu sprechen (natürlich nicht nur). Sie werden auf diese Weise angeregt, hin und wieder über das nachzudenken, was sie „da eigentlich tun“ und wie sie es machen. In dieser Reflexion über das eigene „Machen“ verbirgt sich laut der Hattie-Studie von 2009 ein Lernfaktor mit einer hohen Effektstärke (Lotz/Lipowsky 2015, S. 103).

Der Kontakt und der Austausch innerhalb der Interessensgruppe scheint Beobachtungen zufolge die Stärke einer derartigen Einrichtung zu sein. Wenn sich Schülerinnen und Schüler mit ähnlichen Interessen begegnen und über ihre Ideen sprechen, greift der Wirkmechanismus des „Idea-Sharing“: die Konfrontation mit den Ideen der anderen regt das divergente Denken an.

Ein weiterer nicht zu unterschätzender Faktor dieser informellen Gruppierung ist die motivierende Wirkung. Das lustvolle Nachgehen einer Sache und deren Präsentation in einem Kreis Gleichgesinnter erhält oder steigert die Motivation (Abb. 4).

Abb. 4: Ein Plakat vor dem Eingang zur Jahresausstellung des Arbeitskreises „Kunstclub“ in Bayreuth.

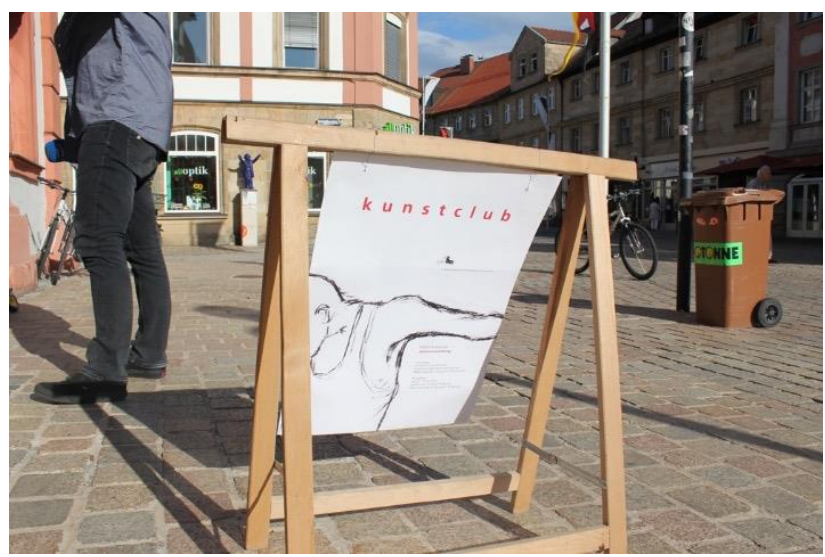


Quelle: Mayer_Vernissage_Kunstclub

Darauf basiert auch die Wirkung vieler Genreseiten im Internet. Leider sind diese oft, was die Kreativität betrifft, eher einengend als öffnend. Heute wird vor allem von der Kognitionsforschung gerade darin ein Indikator und wichtiger Katalysator für das Entwickeln von individuellen Dispositionen gesehen. Eine Schülerin bzw. ein Schüler "will“, sie bzw. er ist motiviert, intrinsisch motiviert. Diese Art von Motivation kann man nicht erzwingen, aber man kann sehr wohl das Umfeld so gestalten, dass sie entstehen kann oder erhalten bleibt. Es kann laut der Kognitionsforscherin Ziegler zu intrinsischer Motivation kommen, wenn man sich selbst als kompetent erlebt bzw. wenn man seine Handlungen als selbstbestimmt und selbst verursacht erfährt (Ziegler 2014, S. 97f.). Dafür bietet ein Kunstclub genügend Möglichkeiten.

Die Arbeit im Kunstclub zeigt, dass es eigentlich einfach ist, intrinsische Motivation aufrecht zu erhalten, dass Sudeln und Schmieren nicht schadet und dass keine allgemeine Zielvorgabe (wie etwa ein Thema) notwendig ist, um individuellen Dispositionen Raum zur Entwicklung zu geben (Abb. 5).

Abb. 5: Zeichnung aus einer Serie von Figuren die auf das Format bezogen sind, selbst gewähltes Motiv einer Schülerin der neunten Jahrgangsstufe aus dem Arbeitskreis „Kunstclub“.

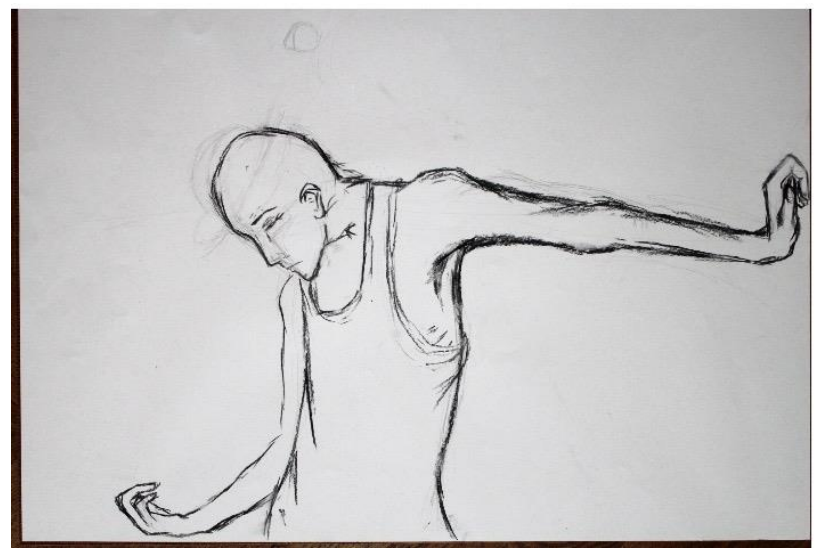

Quelle: Mayer_Kunstclub_Nastassia

Die bisher besprochenen Beispiele zeigen, dass individuelle bildnerische Prozesse im Wesentlichen durch selbstgewählte Tätigkeitsfelder innerhalb eines Themenbereichs oder durch selbstmotiviertes Arbeiten gefördert werden können. Ist entsprechend der Individualisierung der Schülerinnen- bzw. Schüleraktivitäten eine Individualisierung der betreuenden Tätigkeiten der
Lehrperson notwendig und zielführend? Wie wirksam ist eine individuelle Betreuung bei einer von der Schülerin bzw. vom Schüler selbst gewählten bildnerischen Tätigkeit?

Die Universität Augsburg bündelt in einem großangelegten, fächerübergreifenden Projekt Studien zum Umgang mit Heterogenität und zur Begabungsforschung (LeHet 2017). Im Kontext dieser Untersuchung wurde in Kooperation mit dem Lehrstuhl für Kunstpädagogik an der PhilosophischSozialwissenschaftlichenen Fakultät eine Untersuchung an einem Bayerischen Gymnasium durchgeführt.

Auffällig motivierten Schülerinnen und Schülern wurde für den Zeitraum eines Semesters eine Kunstlehramtsstudentin bzw. ein -student als persönliche Mentorin bzw. persönlicher Mentor an die Hand gegeben. Über einen privaten Chat konnten diese die bildnerischen Prozesse der Jugendlichen zeitnah und intensiv betreuen. Theoretisch fundiert wurde die Studie auf den Ideen des russischen Psychologen Wygotski, der in den 1930er Jahren seine Theorie über die „Zone der nächsten Entwicklung" (Wygotski 1956/1987, S. 78ff.) formuliert hatte. Diese besagt, dass Erziehungsmaßnahmen nicht nur von dem aktuell diagnostizierten Entwicklungsstand eines Kindes abhängig gemacht werden sollten, da sie nur ein Bild davon geben können, wie die Entwicklung bisher verlaufen ist, nicht aber wie sie im Moment läuft oder wohin sie geht. Es gehe hingegen vielmehr um die Richtung, welche die Entwicklung nehmen wird. Er nannte dies „die Stufe ihrer nächsten Entwicklung" (Wygotski 1956/1987, S. 78ff.). Diese Stufe erreichen Lernende eher, wenn sie statt alleine zu arbeiten, von kompetenten anderen unterstützt und angeregt werden. Das geschieht durch Konfrontation mit Impulsen und Ideen, die etwas über ihrem aktuellen Wissenstand liegen, also „minimalen didaktischen Hilfen“ (Lotz/Lipowsky 2015, S. 160 u. 180).

Zu Beginn des Semesters wurden den Studierenden Mappen der beteiligten Schülerinnen und Schüler vorgelegt. Auf dieser Grundlage wählten die Studierenden ihre Mentee aus und traten mit ihnen in Kontakt. Die Schülerinnen und Schüler begannen alsbald, ihre Werke zu posten und sich mit den Mentorinnen und Mentoren über ihre Gedanken auszutauschen. Es ging um Themen, Techniken und Inhalte von Zeichnungen, Skizzen und Basteleien. Der Fokus für die Mentorinnen und Mentoren lag allerdings nicht auf konkreten Korrekturen der bildnerischen Arbeiten, sondern auf 
der Überlegung, welcher Entwicklungsschritt sich aus der diagnostizierten Situation erschließen lasse. Darin lag ein nicht zu unterschätzendes Problem für die Studierenden, die sich dabei weder an ein Regelwerk, noch an Vorlagen orientieren konnten. Die Grundlage für die wiederholten Mikrodiagnosen und vor allem für die daraus resultierenden Impulse lagen, so der einhellige Ton der Mentorinnen und Mentoren, in der eigenen bildnerischen Erfahrung.

Die Schülerinnen und Schüler fühlten sich durch die Arbeit mit den Mentorinnen und Mentoren grundsätzlich motiviert, angehalten auch Neues auszuprobieren, und sie vertieften die Reflexion über ihr bildnerisches Tun. Es stellte sich fast bei allen ein Gefühl ein, sich weiterentwickelt zu haben (Abb. 6, 7, 8).

Abb.6: Zeichnung eines Studentischen Mentors der Universität Augsburg bei dem gemeinsamen Arbeitstag mit ihren Mentees.

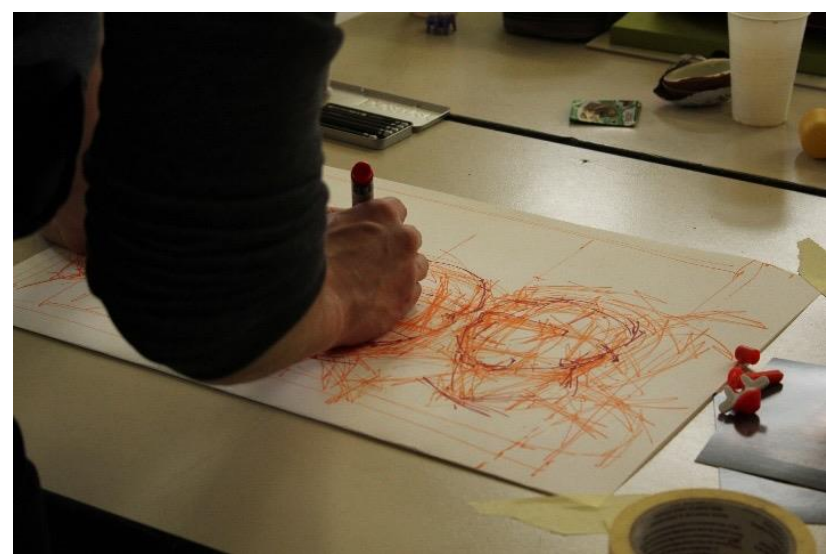

Quelle: Mayer_Mentorenprojekt

Abb. 7: Zeichnerische Auseinandersetzung eines Schülers mit dem Inhalt eines Überraschungseis: Thema eines gemeinsamen Arbeitstages der Mentoren/-innen mit ihren Mentees.

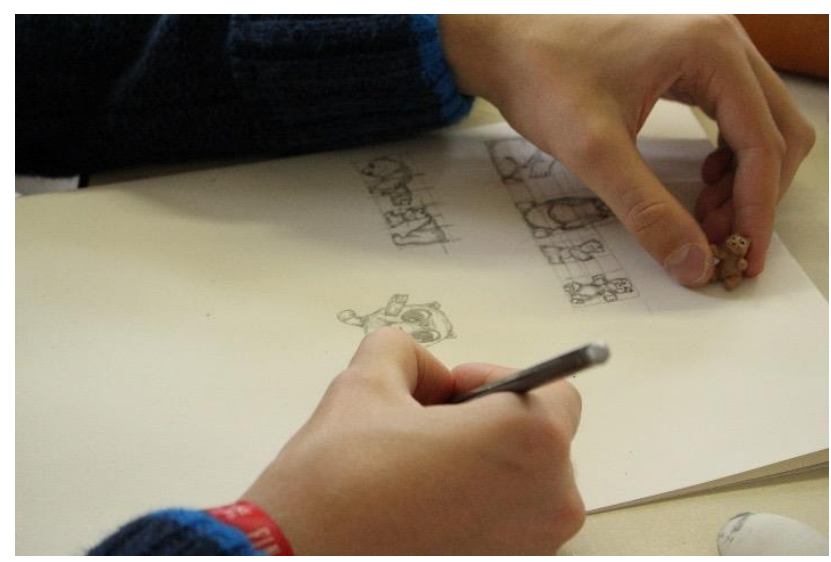

Quelle: Mayer_Mentorenprojekt
Abb. 8: Weiterentwickeln eines Motivs aus einem Überraschungsei. Eine Bayreuther Schülerin beim gemeinsamen Arbeitstag mit ihrer Mentorin.

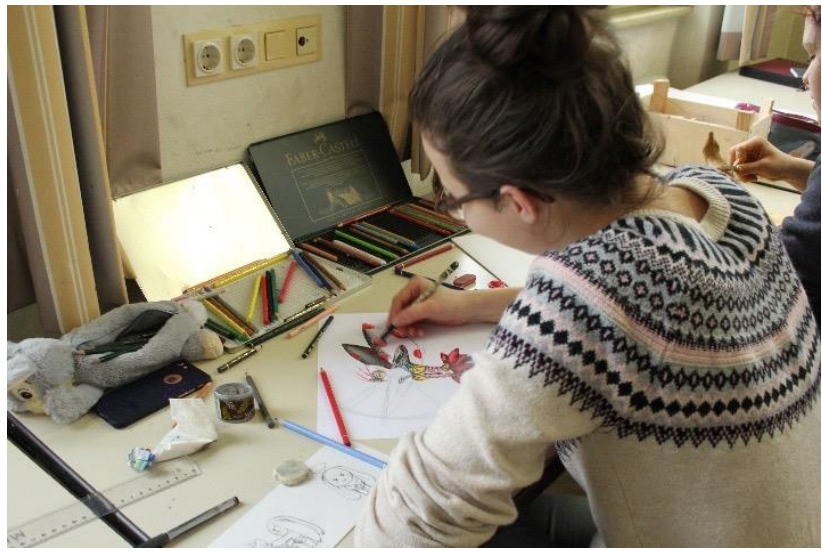

Quelle: Mayer_Mentorenprojekt

Grenzen bot bei fortschreitender Zeit das Onlinemedium, der virtuelle Kommunikationsraum. Die dadurch mögliche potentiell permanente Kommunikation wurde hingegen nicht als entscheidender Vorteil gesehen. Das Fehlen eines persönlichen Kontakts und damit der Möglichkeit, Tonfall und Mimik wahrzunehmen, wurde bei der immer tiefer gehenden Konversation ein entscheidendes Hindernis. Für eine effiziente individuelle Förderung, so könnte man daraus schließen, ist eine persönliche Präsenz unumgänglich (Abb. 9, 10, 11). Was sich aber sehr positiv auswirkte, war die individuelle Betreuung über einen längeren Zeitraum hinweg. Man könnte hier eine Bestätigung der Relevanz einer kontinuierlichen langfristigen Betreuung sehen.

Abb. 9: Organische Formen aus Ameisen entwickelt; Aufhängen der Gemeinschaftsarbeit einer Mentorin und ihrer Schülerin im Schulkorridor.

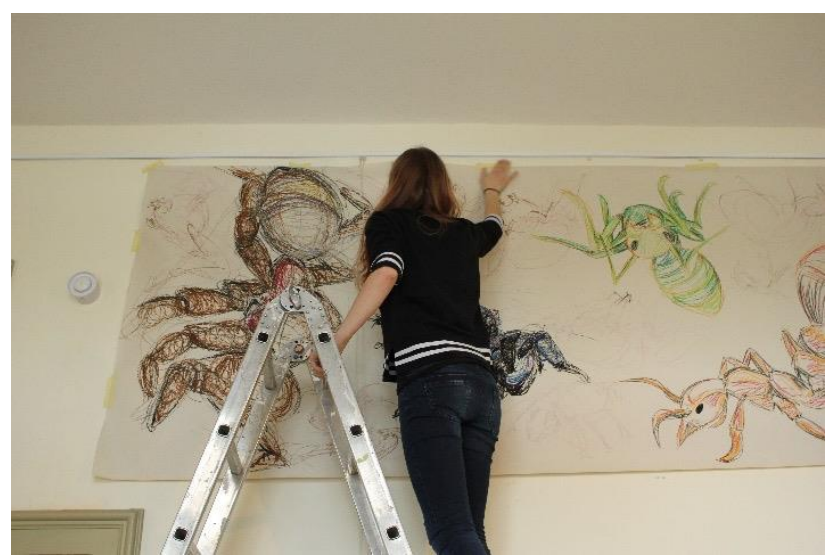

Quelle: Mayer_Mentorenprojekt 
Abb. 10: Arbeitsplatz Fußboden, Entwickeln von fantastischorganischen Formen, Gemeinschaftsarbeit einer Mentorin mit ihrer Schülerin.

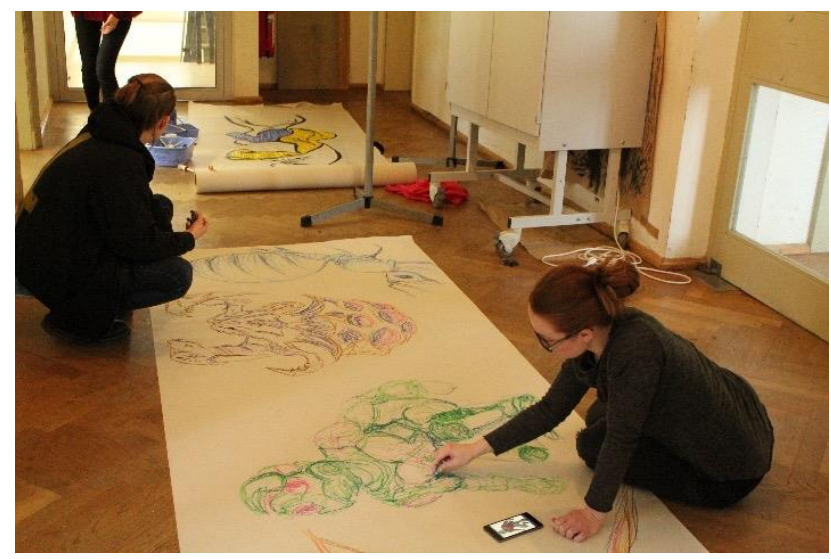

Quelle: Mayer_Arbeitssituation_Mentorenprojekt

Aus dem Mentorenprojekt kann man zusammenfassend schließen, dass eine Förderung bildnerischer Prozesse also nicht unbedingt synchron oder zeitnah ablaufen muss, dafür aber langfristig und persönlich begleitet sein sollte.

\section{RESÜMEE}

Es ist deutlich geworden, dass eine individuelle Förderung bildnerischer Prozesse eine dem Kunstunterricht innewohnende Funktion ist. Sie ist auch im Regelunterricht möglich und beginnt zum Beispiel schon dann, wenn eine Schülerin bzw. ein Schüler eine Sonderlösung vorschlägt oder einen eigenen Bereich bearbeiten will.
Eine Möglichkeit individuelle Veranlagungen und Motivationen unter einen Hut zu bringen, ist eine offene Aufgabenstellung. Offene Aufgaben, regen - so auch die Hattie-Studie (Lotz/Lipowsky 2015, S. 103) - zu divergentem Denken an und bieten mehr Möglichkeiten mit persönlichen Veranlagungen an die Bearbeitung heran zu gehen.

Ein probates Mittel dafür, das allerdings immer weniger eingesetzt wird, ist die echte Projektarbeit mit Interessensgruppen statt Altersgruppen. Weitgehend selbstbestimmtes Arbeiten auf vielen Tätigkeitsfeldern ermöglicht hier das Einbringen individueller Leistung in soziale Interaktion. Auch ein gezieltes Provozieren von Querdenken, von eigenen Lösungen, von Änderungen in den Rahmenbedingungen gehören dazu, stets begleitet von einer Reflexion des eigenen Tuns, das verbalisiert und im Team kommuniziert werden muss.

Für eine Individualisierung bietet der Kunstunterricht viele Gelegenheiten. Die Lehrerin bzw. der Lehrer ist in individualisierten kunstdidaktischen Konzepten nicht Reisender in einer Lernlandschaft oder ein im Hintergrund stehender Coach, sondern ein individueller Mentor für alle Schülerinnen und Schüler einer Klasse. Er betreut jeden gezielt durch Einzelgespräche und versucht mit minimalen Impulsen die Stufe der nächsten Entwicklung anzustoßen - und das in persönlichen Kontakten über Jahre hinweg.

Auf diese Weise kann eine effektive Förderung im Regelunterricht stärker berücksichtigt werden, ohne die Lehrkraft durch zusätzliche Aufgaben zu überfordern.

\section{LITERATURVERZEICHNIS}

Brodbeck, Karl-Heinz. Neue Trends in der Kreativitätsforschung. In: Psychologie in Österreich, $4 \& 5,2006$, S. 246-253.

Hecht, Martin: Das Drama um das hochbegabte Kind. In: Mehlhorn, Gerlinde/Schöppe, Karola/Schulz, Frank (Hg.): Begabungen entwickeln und Kreativität fördern. München (kopaed) 2015, S. 21-34.

Kerschensteiner, Georg: Die Entwicklung der zeichnerischen Begabung. München (Gerber) 1905. Kirchner, Constanze: Ästhetische Bildung im Fach Kunst der Primarstufe. 2008. In:
http://docplayer.org/33457447-Aesthetischebildung-im-fach-kunst-der-primarstufe.html

LeHet - Lehrerprofessionalität im Umgang mit Heterogenität, Universität Augsburg. 2017. In: https://www.uni-augsburg.de/projekte/lehet/

Lotz, Miriam/Lipowsky, Frank: Ist Individualisierung der Königsweg zum erfolgreichen Lernen? In: Mehlhorn, Gerlinde/Schöppe, Karola/Schulz, Frank (Hg.): Begabungen entwickeln und Kreativität fördern. München (kopaed) 2015, S. 155-219

Lotz, Miriam/Lipowsky, Frank: Die Hattie-Studie und ihre Bedeutung für den Unterricht. In: Mehlhorn, Gerlinde/Schöppe, Karola/Schulz, Frank (Hg.): 
Begabungen entwickeln und Kreativität fördern. München (kopaed) 2015, S. 97-136.

Luther, Michael: Das große Handbuch der Kreativitätsmethoden. Bonn (managerSeminare Verlags $\mathrm{GmbH}) 2013$.

Miller, Monika: Zeichnerische Begabung. Indikatoren im Kindes- und Jugendalter. München (kopaed) 2013.

Proetel, Tim: Referat Kunst/Theater und Film. Staatsinstitut für Schulqualität und Bildungsforschung, zum LPP. E-Mail an den Autor vom 15.04.2016 (unveröffentlicht).

Penzel, Joachim: Aufgaben stellen. In: http://www.integrale-

kunstpaedagogik.de/assets/ikp_um_aufgaben_stel len-i_penzel_2016.pdf

Peters, Maria: Im experimentellen Zeichnen gehen die Namen der Dinge fremd. In: Kunst+Unterricht, 223/224, 1998, S. $15 f f$.

Roth, Gerhard: Begabung und Hochbegabung. In: Mehlhorn, Gerlinde/Schöppe, Karola/Schulz, Frank (Hg.): Begabungen entwickeln und Kreativität fördern. München (kopaed) 2015. S. 55-78.
Lehrplan+. In: http://www.lehrplanplus.bayern.de/fachprofil/gy mnasium/kunst

Rabe, Thies: Sprecher der SPD Kultusminister, auf der Kultusministerkonferenz am 28.11.2016. In: https://www.kmk.org/fileadmin/Dateien/pdf/Press eUndAktuelles/2016/2016-11-

28_Gem.Initiative_Leistungsstarke-Beschluss.pdf Schulz, Frank: Das Problem der bildnerischen

Begabung als Aspekt der Kreativitätsentwicklung. In: Mehlhorn, Gerlinde/Schöppe, Karola/Schulz, Frank (Hg.): Begabung entwickeln und Kreativität fördern. München (kopaed) 2015, S. 405-466.

Wygotski, Lew Semjonowitsch: Das Problem der Altersstufen und die Dynamik der Entwicklung. In: Ausgewählte Schriften. Band 2. Moskau 1956, Köln 1987, S. 78-90.

Ziegler, Esther: Talent und Begabung in der Kognitionspsychologie. In: Stamm, Margrit (Hg.): Handbuch Entwicklungspsychologie des Talents. Bern (Huber) 2014, S. 97-106

MAYER, Stefan. Individuelle Förderung bildnerischer Kompetenzen im Kunstunterricht. JOSSHE: Journal of Social Sciences, Humanities and Research in Education. v. 3, n. 2, p. 1-7, jul./dez, 2020. DOI https://doi.org/10.46866/josshe.2020.v3.n2.94 